\title{
"Snake": One More Terramorphic Entity of the Hypothetical Venus Fauna
}

\author{
Leonid V. Ksanfomality \\ Space Research Institute of the Russian Academy of Sciences, Moscow, Russia
}

E-mail address: ksanf@rssi.ru

\begin{abstract}
During the years that passed since the time of TV experiment plo, VENE A landers (1975 and 1982), no similar experiments or missions to Venus b been per ned. Analysis of treated once gain VENERA-13 and VENERA-14 panoramic imag st to (amon other hypothetic living entities) a 'snake' object about $40 \mathrm{~cm}$ in size possessi o ppare rramorphic features. The snake's body stands out with its honeycomb, spotty surfo ayuinst the /plates close by. The 'snake' can be included into the list of the most signif cant findings of the hypothetical Venusian fauna. Apart from that, of interest is a 'dove' object, lthough deta ls of its structure cannot be discerned. The snake's body show slow movements, whic another e idence of the Venusian fauna's very slow style of activity, which appears to be associate h itc nergy constraints or, and that is more likely, with the properties of an internal $1 m$ of its vody. The terramorphic features of the fauna, if they are confirmed, may point out at itstar. laws of the animated nature.
\end{abstract}

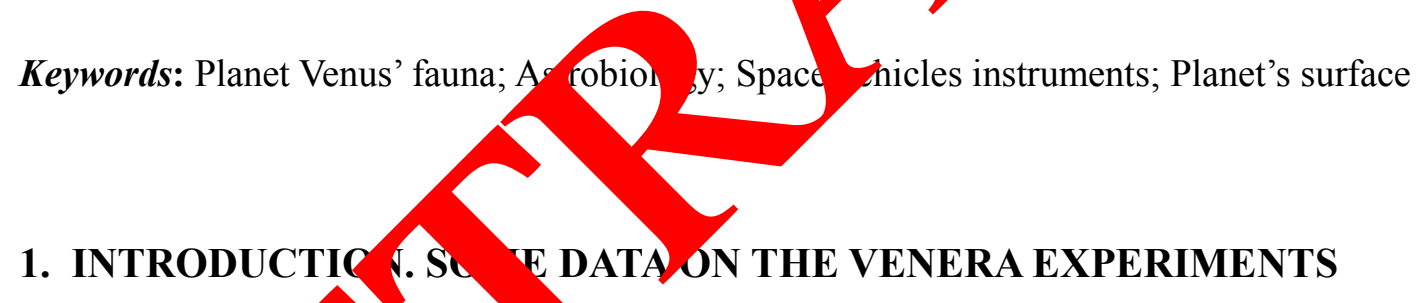

On Marc 1 an 15,1982 , periments in television photography instrumented by the landers Ven 13 , 14 yielded 33 panoramas of the Venus surface (or their fragments) at the landing sil he met odology of the television experiments on the surface of Venus and the da list on ex perimental data have been discussed in detail in [1-3] and are not rep ted hy e.

the pas years, no similar missions have been sent to Venus. In connection with the renewed cest in what was occurring during the experiment and to the discovery of manifestations of possible life revealed on some of the pictures, the panoramas were re-examined. A train of papers [2-4] published in 2012 presented some data on the hypothetical Venus fauna that survives under physical settings that are radically different from the Earth's.

Along with unfamiliar forms, some of the found objects are closely reminiscent of the forms of some of Earth's living organisms. The phenomenon is called terramorphism. 
E.g., the term might be referred to as a 'bear cub' [4], as found in a VENERA-9 panorama (Fig. 1). The resolution in the panorama is 21 ', and for that reason, despite the bear cub's position, the identifiability of finer details is limited.

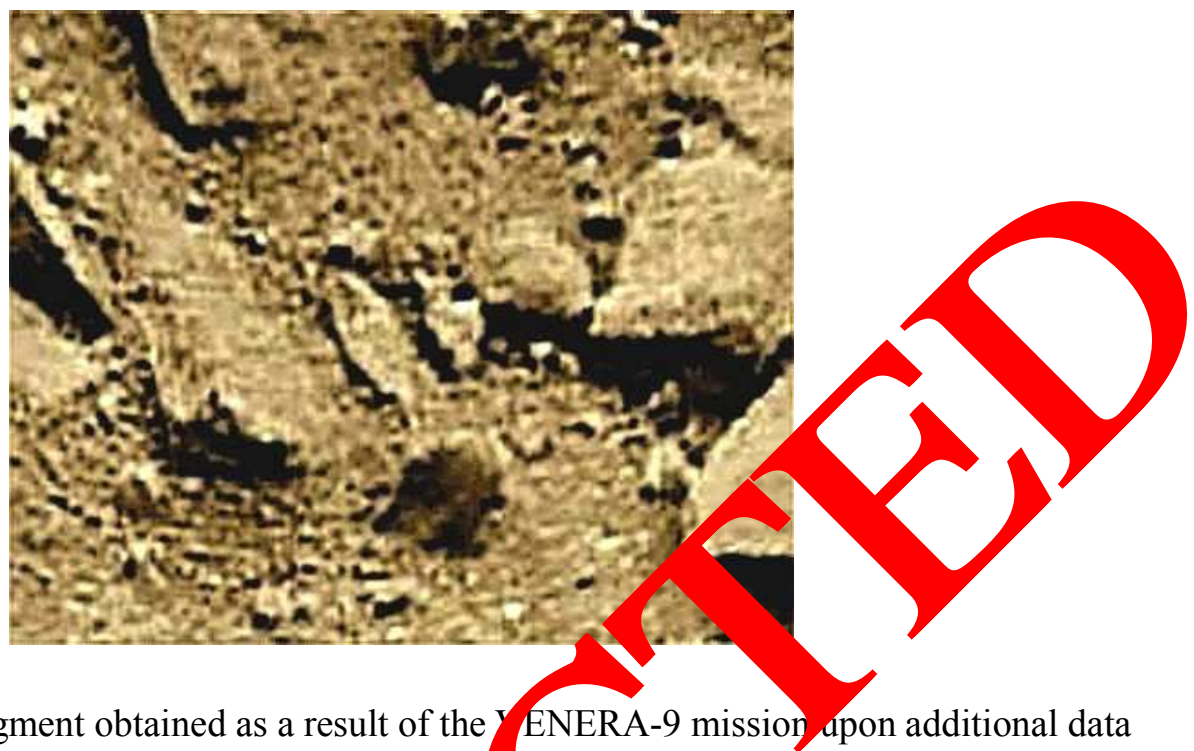

Fig. 1. Panorama fragment obtained as a result of the processing. In the forefront, there is a small object seen contours, the object is distinguished from the sharp stone wes. To the eft beyond the object, over the ground, there propagate long furrows that terminate und Bear. Apparently, these tracks indicate the preceding motio the object.

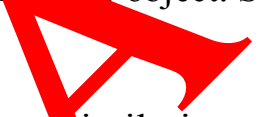

Still more pronounced terrar ism, or imilarity to the Earth's fauna, can be found in the 'snake' whose features ar discu sed in th paper. The phenomenon of terramorphism, which is observed on anotbre tally different natural settings, in case it is confirmed, can note as et unkm laws of living form development and can render

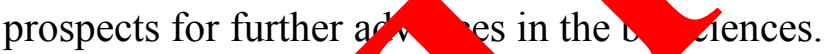

As noted in ea er $p_{4}$ shed papers $[2,5,6]$, during the years that passed since the time of TV experimer that empl the VENERA-9, -10 (1975) and VENERA-13, 14 (1982) landers, no si alar e, veriments, ave been performed. Papers [2-6] addressed the findings of revision ana reatm nt as well as the analysis of the VENERA-9 and VENERA-13 panoramic imag Going arther than the publications mentioned above, this paper offers the result on new a of VENERA-14 lander panoramas, in which new interesting and un fir fings wer revealed.

ascussed in this paper drew researchers' attention before (in 1983) due to the unc non geological features of the region [7]. The objects of hypothetical fauna that were foun at both landing points (VENERA-13 and VENERA-14) are different (with a single exception), and the differences could be related, in a way, to the features of the landing sites.

The coordinates of the VENERA-14 landing site are $13^{\circ} \mathrm{S} / 310^{\circ} \mathrm{E}$, with a site altitude of $1.3 \mathrm{~km}$ above the $6051 \mathrm{~km}$ radius level and a temperature of $738 \mathrm{~K}$ and pressure of $9.47 \mathrm{MPa}$. A fragment of the area map (in the northern part of Panina Patera) is shown in Fig. 2. 


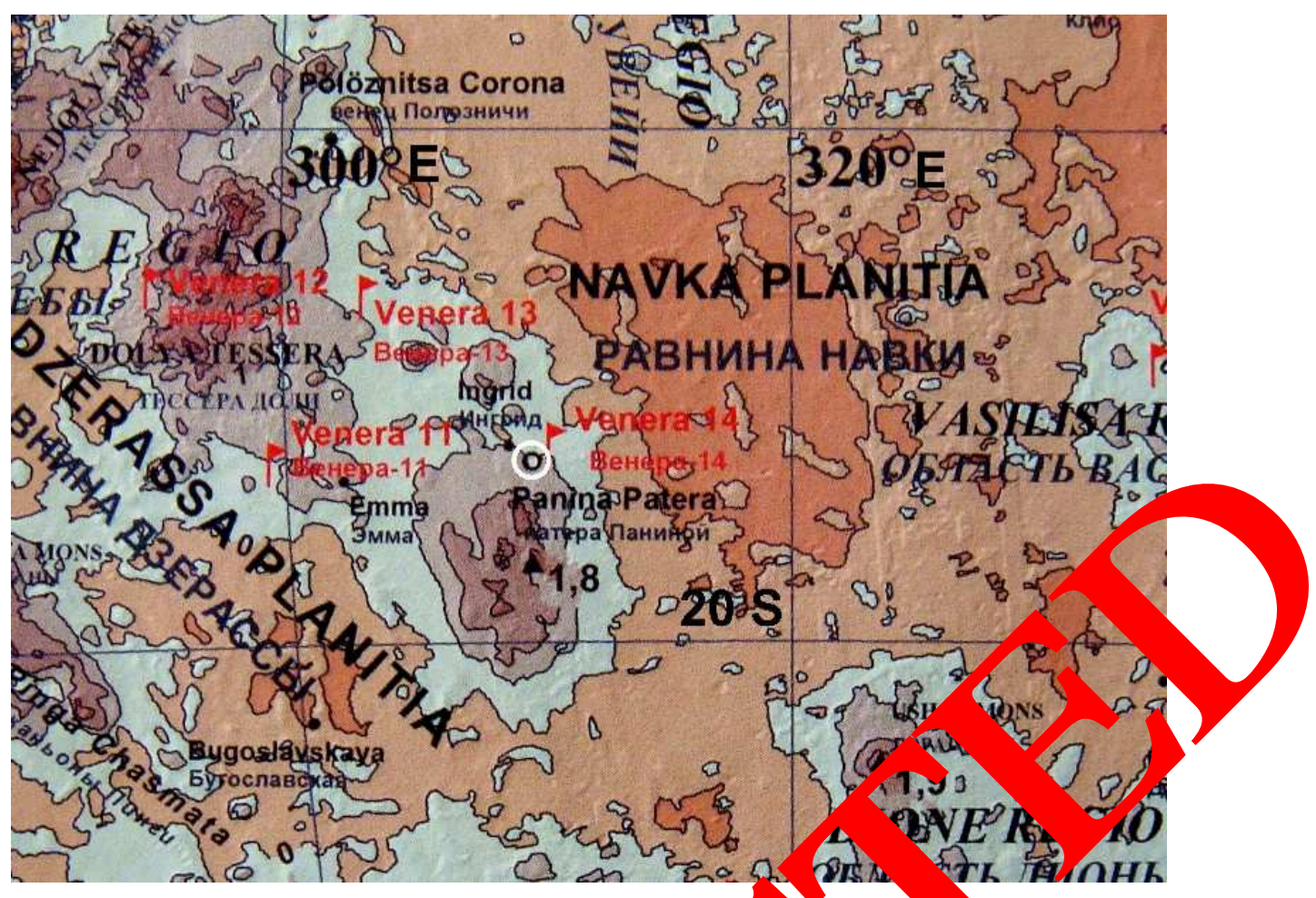

Fig. 2. A fragment of the Venus surface map [8]. The wh e circle denotes th VENERA-14 landing site. At the Venus equator, $1^{\circ}$ of longitu /latitude equals $105.6 \mathrm{~km}$.

It is essential to give some data on alanet strme properties at the VENERA-14 landing site. The geological features of the rear of the VENERA-13 landing site. The planet surface t ne siv, is a flat plain covered with a layer of fractured high-density (seemingl fied) $\mathrm{n}$ terial that has clear boundaries of separate plates (Fig. 3).

The mechanical prope fes studied by [9] in a special experiment. The material is of high load-b aring ca ity that is 25 times greater, according to [9], than that of the loose ground at the VERA-13
of basalt or granite.

\section{AN EXTI VECOS B DDY ON THE SURFACE OF THE STRATIFIED ROCKS} Kosmicheskiye Issledovaniya published in 1983, directly after the
the a
'large st ase shelves $1-2$ to $5-10 \mathrm{~cm}$ high. ... The layers are different from each other in
their refled
close to the TV camer.' Then, the researchers focused on an unusual surface area that was

They wrote, 'Apart from the clearly stratified rocks, some of the panorama sections show rock outcrops without layering. For example, in the V14-1 panorama, to the right of the color test end, in a shallow depression of the microrelief, one can see a relatively dark, spotty, honeycomb surface with an odd zoned orientation of the spots. It appears that, from under the unconformable layers of the foliated unit, a rock surface of a different structure peeps out.

This rock could underlie the foliated unit or make up some 'extraneous' entity within the foliated unit. 
At the left-hand boundary of the V14-2 panorama, there are some spotty shapes that might be outcrops of similar spotty rocks as well' [7], 1983, p. 345). It remains to admire the insights of the K.P. Florensky team. In a recent publication [10], the author of this paper notes the other 'spotty shapes in the V14-2 panorama' as possible Venusian flora objects, although it is rather difficult to underpin this hypothesis.

At the same time, the 'extraneous spotty, honeycomb entity at the V14-1 panorama' could have been the most amazing finding of K.P. Florensky et al. Repeatedly, they come back in their paper to the 'small outcrops of spotty rocks.' As is shown below, it only remains to regret that they failed to get further into research on that small 'extraneous entity' which would have been quite worthwhile.

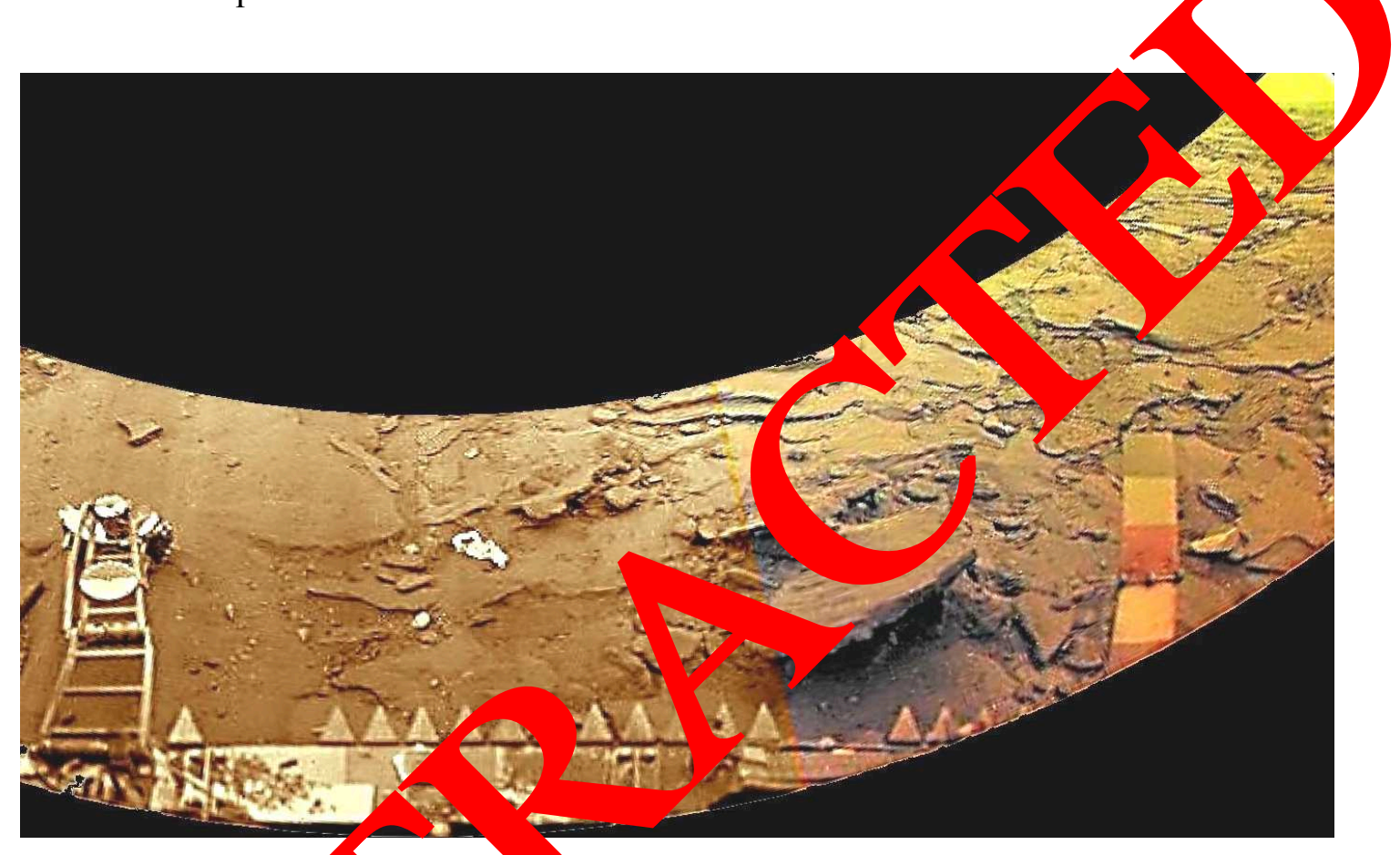

Fig. 3. VENERA-14 $p$ non 1-BW1. e staircase truss (at left) is an unrolled lever system of the PrOP device that mea res th d-bearing capacity of the ground. The unrolled truss is $60 \mathrm{~cm}$ long. In ght-hand part: color control strip.

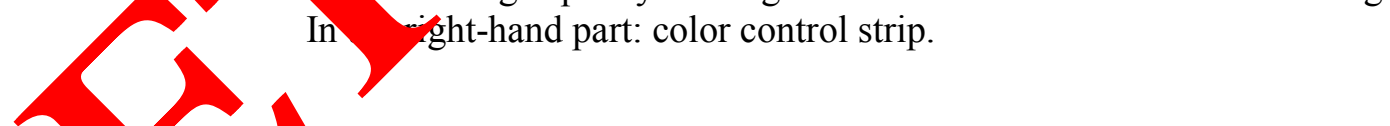

With respe of the ossment [9] of a rather high load-bearing capacity of the ground, the y iter f the $\mathrm{pa}_{\mathrm{a}}$ ] ] comment that the capacity is greater than the estimate based on the $\mathrm{dy}$ ic $\mathrm{p}$ rameters of the lander impact while landing, and they assume that the earlier assess 1 ts mung. If they are correct, then the error occurred for a reason that was difficult to foreso Similar to in all other VENERA landers, the front windows of the TV cameras were cover $d$ with caps that had to be blasted off after landing.

The caps were removed, and one can see in Fig. 3 that the PrOP-measuring cone was exactly on a cap (the white semi-cylinder $20 \mathrm{~cm}$ in size, see Fig. 3). At the same time, the authors of [9] noted that the measuring cone proved to be beyond the cap; thus, the measured data were valid because they had to be related to the ground rather than to the cap. 


\section{VENERA-14 TV-PANORAMAS THAT CONTAIN 'EXTRANEOUS BODY' IMAGES}

The objects found in the panoramic images from VENERA-14 draw upon the sequences of images that are organized in a way that is different from the earlier considered VENERA13 panoramas [2-5]. The first sequence was designated Series 1. Its data are almost entirely the same as in Series 6, which is evidenced by practically identical positions of the dot interferences. Series 9 repeat scanning was reproduced in Series 13 in accordance with the same schedule, but the data were different from Series 1 and 6 in both the position of the interferences and the small shifts in the scene objects.

First, full-scale $\left(180^{\circ}\right.$ each) black-and-white panoramas were scanned thin the 4 $750 \mathrm{~nm}$ spectral range) by camera V-14-1 (designations as in [2,3,5], having 945, 998 lin in 1/6 and 9/13 sequences, respectively. Afterward, only the following s/ort image rme its were transmitted: red (590-720 nm), green (490-610 nm) and (useles blue, 330-3. lines each and, then, an incomplete $\left(118^{\circ}\right)$ black-and-white panorama $W$ the en of each sequence, there were full-scale BW-3 panoramas again, of 991 a d 994 lin

The time for one line of scanning was $780 \mathrm{~ms}$, and th a comple sanorama was approximately $13 \mathrm{~min}$ [1]. Considering the observed lever ff no, the only red fragments that are acceptable for analysis belong to the $1 / 6 \mathrm{~s}$ qua e. The fragments can be treated in two series. The BW-2 panoramas do not ontain the object/ander study. The noise level in panorama $1 / 6 \mathrm{BW}-1$ is small (Fig. 3), wh the $1 / 6 \mathrm{~B} / \mathrm{V}-3$ panorama has noise all over. In the later panoramas $(9 / 13 \mathrm{BW}-1$ and $9 / 13 \mathrm{~W}-3)$, the noise level gradually rises, although the object under study, however-strange th oe, remains almost noise-free (which was considered in [3]). Later, the $1 / 6$ nanorama was substantially improved.

It is worthwhile to recall some data on he the experiment [1]. Two optomechanical cameras were installed on opposite sides of the lander. (This paper considers only the data that was received from amo 1 of Vh VERA-14.) The camera pupils were at $0.9 \mathrm{~m}$ above the land surface, and the houndar of the panoramas in the sector under study was approximately $3 \mathrm{~m}$ fro the axes of the scanning cameras were at $50^{\circ}$ to the vertical [1], whi made it $\mathrm{p}$ ible to resolve 2 millimeter details of the surface in immediate proximity o ander an approximately $10 \mathrm{~m}$ at the mathematical horizon (at $3.3 \mathrm{~km}$ over the en surfac Vllumination (with scattered sunlight) was 3-3.5 klx [11], and the solar zenith angle during the periment was $36^{\circ}$.

As has ready en noted in [5], the landers operated much longer than was stated in [11]. The mato given low cover $1 \mathrm{hr} 33$ min of VENERA-14 operations on the planet surface data re rerenced to time by using the transmitted line number contained in the ouse eping $p_{y}$ of the experimental data. The angular resolution is 11'. Each line com s pixels and 41 elements of housekeeping data. To wait until the dust settles, minute delay after landing was introduced before the cap was blasted off and transmiss, first the firsorama was commenced.

\section{4. 'EXTRANEOUS HONEYCOMB ENTITY'}

The panorama fragments given below, similar to in the other papers of the series [2-6], have a limited resolution, only 11 ' per pixel, which is why one must be alert and patient while working with these images. The 'extraneous honeycomb entity' (according to [7] terms) is in the rightmost part of Fig. 3, to the right from the color control panel. It might be useful to 
mention that the panel is $10 \mathrm{~cm}$ wide, and each color field is square. The depression with the 'extraneous honeycomb entity' inside is approximately $20 \mathrm{~cm}$ to the right from the panel. Fig. 4 (frames 1-4) shows four untreated fragments of independent primary images of the object. Compared with the original images, the brightness and contrast of the images in Fig. 4 have been leveled. The 'extraneous entity' is in the center of each shot. The signal-to-noise ratio in the black-and-white panoramas is more than sufficient and gives a chance to use some of the primary images as they are.

Their treatment is required only for further operations. Notwithstanding, the noise-tosignal ratios in the single-colored images are insufficient for a clear picture; as a result these fragments were used exclusively for composite color image synthesis. Co imonly, stacking of repeat original images allows us to improve the identify ability the deta However, an attempt to stack the pictures of the 'extraneous honeycomb entity' in a number of cases, depending on the choice of the stacking pilot poin, when son art of the pictures are aligned, some other parts become slightly out of focus

The reason behind the alignment problem proved to be in $\mathrm{sma}_{\mathrm{a}} \mathrm{ft}$ in $\mathrm{tl}$ bject's position. Inasmuch as there are no less than four black-and-wh e images t are well suited for analysis even in their original quality (panoramas BW 1/ M of sequen $1 / 6$ and 9/13) and no less than three same color-separated images, one car nanas produce a sequence of certain fragments as conventional 'snapshots' of the sar cara (Fig. 4,

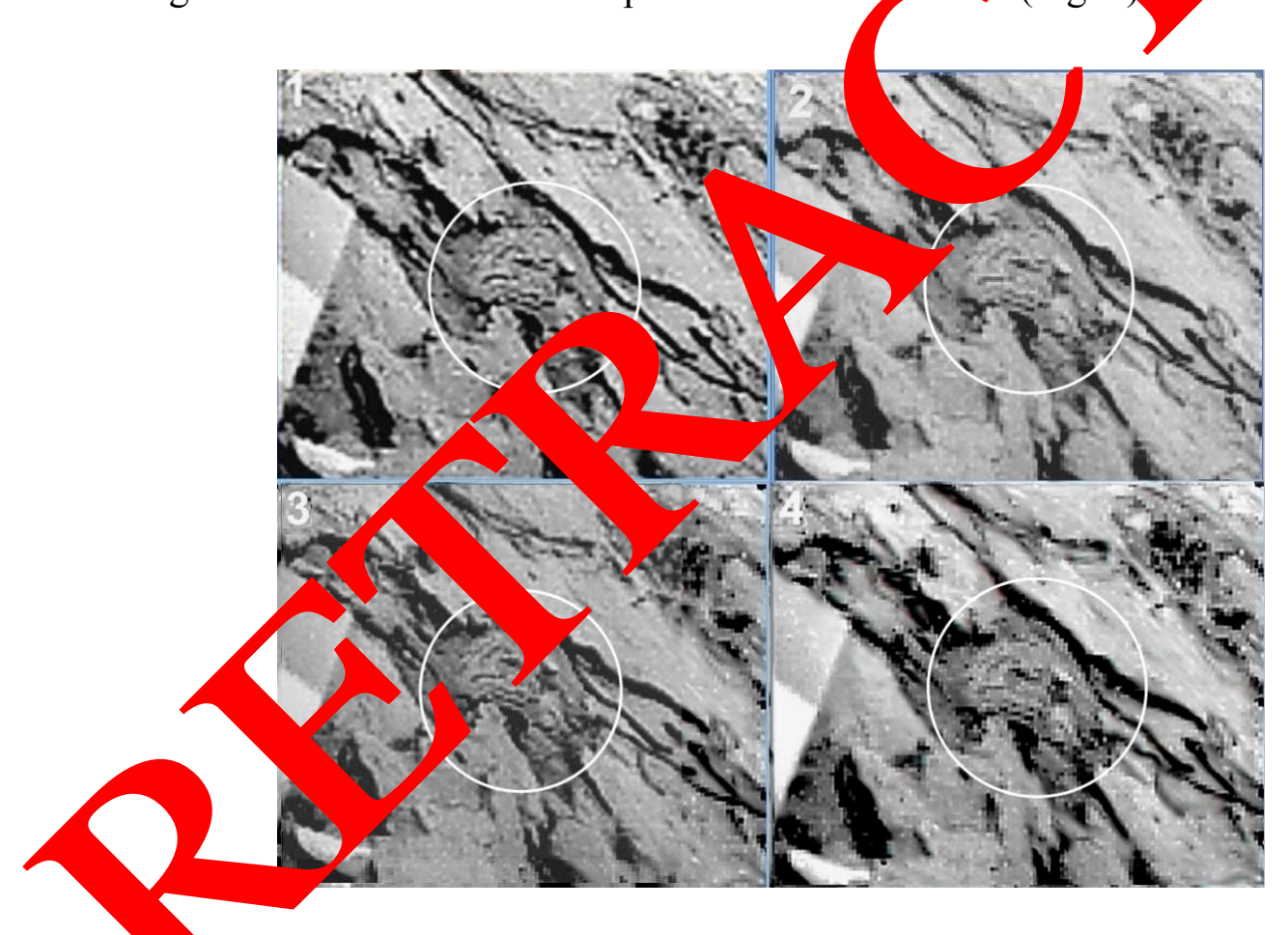

Fig. 4. Msecutive shots of the 'extraneous honeycomb entity' in the source black-and-white panoramas of VENERA-14 camera 1: 1 . file BW-1, sequence $1 / 6 ; 2$. file BW-3, sequence $1 / 6 ; 3$. file

BW-1, sequence $9 / 13$; 4. file BW-3, sequence $9 / 13$. The shots are 34,30 and 32 min apart, respectively.

During the operational period, due to the continuous rise of the temperature in the optic and electronic parts of the camera, the resolution varied: first, it became worse (shots 1-2, Fig. 3.3); then, it improved (3); and then, it deteriorated again (4). 
The instants when the transmission of each fragment in Fig. 3.4 commenced, starting from the beginning of the shot 1 transmission, were $2044 \mathrm{~s}(34 \mathrm{~min} 04 \mathrm{~s}), 3874 \mathrm{~s}(1 \mathrm{hr} 04 \mathrm{~min}$ $34 \mathrm{~s})$ and $5792 \mathrm{~s}(1 \mathrm{hr} 36 \mathrm{~min} 32 \mathrm{~s})$. Comparing the transmission instants according to the line numbers, one can see that they do not completely match the table that is given in the express publication [1], while the content of the last panoramas was included in the table without any reference to time at all.

The last line of the 9/13 BW-3 panorama, from which shot 4 was taken, refers to the instant of $1 \mathrm{hr} 49 \mathrm{~min} 27 \mathrm{c}$. Similar to in the case of VENERA-13, the cited data and the time references of the later series (Nos. 9 and 13 for VENERA-14) were revised further on [12]

Fig. 5. The image of "sn? made of st. $\mathrm{dBW}$ shots (except BW 3). For better viewing, the image is turned $38^{\circ}$ relative t Fig. In the inser below, the arrows indicate the hypothetical structure of the snake as it can be raved: he head with an eye; 2 - the coiled body of the snake; 3 - the crest;

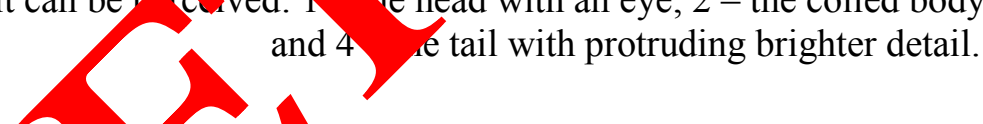

Th- humar ision capability of generalizing the details of unclear images is very dive in ifferen sons. In astronomy, there is a well-known classical case of dispute be n ovanni Schiaparelli, who managed to compile a map of Mars based on poorly visibl tcres, wd Asaph Hall, who observed Mars visually and discovered two small Mars satellites, aph Hall absolutely could not agree with Schiaparelli's drawings.

The i lage content of the 'extraneous honeycomb entity' in Figs. 4 and 5 was tentatively discussed by the author with his colleagues. Approximately $50 \%$ of them were able to see a coiled snake in the pictures, but the others were unable to notice it, even when it was distinguished with a circle around it as in Fig. 5. With due regard to the aforementioned problems in image stacking, the shots that were prepared to be stacked allowed us to improve the image. Shot 4 of Fig. 4 was not included in it. Fig. 5 shows a treated image of the 'extraneous honeycomb entity.' Similar to in [2-6], no retouch was made whatsoever, except in the insert miniature at the bottom of Fig. 5 for display purposes, where the 'extraneous entity' contours are slightly highlighted. 
It is clear from Fig. 5 that the regularly positioned spotty 'cells' cover the object's surface. The object is in a small depression (5-10 cm deep), and it resembles a coiled snake. Moreover, it appears to possess certain terramorphic features. Conventional (provisional) names that have been suggested for the objects found on the Venus surface remain conventional; at the same time, the name 'snake' is the most practical for the object under study precisely because of its terramorphic features.

\section{THE SNAKE'S STRUCTURE AND MOTION}

To estimate the snake's size, it is convenient to use the color control pan seen in Fi 3 and 4). The height of each field is $10 \mathrm{~cm}$; however, at the distance wher the ke is, $t$ scale is $30 \%$ greater. The overall length of the snake amounts to approxi ately 400

Apart from the snake, one can see some other unusual objects ir igs. 5 d 6 . h ne 9hour direction from the snake, on a flat bright stone, there is a dar $\mathrm{ob}$, pproxi ately 5-6 $\mathrm{cm}$ in size that was conventionally called a 'dove.' Its small di nensions not crmit us to identify any other details apart from the shadows under the $b / a$, under orotruding left part. Because of the $50^{\circ}$ inclination of the scanning camera xis, $t$ urface in Fig. 4 appears to be sloping. The fragment was turned to improve the display comparing the sizes more convenient.

The geometric distortions in Figs. 5 and 6 hav not been eli ninated; however, the angle covering the picture is small $\left(34^{\circ}\right)$ and, as a result, th istortions are not essential. Although a movement is insignificant, the snake's position graduah from shot to shot.

The consecutive positions of the 'extr. antity' in the three sharpest panoramas are presented in Fig. 6. Close examination of th sh is ws us to see gradual, although very small, shifts in the relative position of the si ke's parts (the body, head and tail) and their positions relative to other parts of snake well as relative to the stone plates of the surface. It is more convenie fol ow the s ifts by using the Gif Animator software (a computer analogue of a bli con atu, .....stronomical tool).

It is possible that 'dove' s. as well. However, it does not appear to be feasible to show in this paper th smifts by a ning. As was noted in [13], whenever some shifts of the hypothetical $y$ noun fan revealed, they are always extremely slow, no more than 1 $\mathrm{mm} / \mathrm{s}$. Comparig all of the sh of the sequence, one can arrive at the same or a lower rate for the relati shift the snake's parts. As has already been mentioned, the resolution in the images was lin by the ngle of 11', which is why the identify ability is constrained even in the Fragh ts th at were treated by using 'unsharp mask' processing.

In th 'green ctures (not included here), the snake's body appears to be slightly big th ' the flack-and-white images. One can assume the snake's structure as in Figs. $5-6$, w the snake's hypothetic structure is presented and some parts of the 'extraneous honeycon antity' are indicated. The rightmost brighter part, the 'head' (arrow 1, Fig. 5), faces the cimera with its round 'eye' (the bright round spot can be noticed in any shot). The position of the spot leaves no doubt whatsoever as to its function. The 'head' is approximately $6-8 \mathrm{~cm}$ in size and is reminiscent of the heads of many Earth beings (e.g., birds, reptiles and some marine animals). In Fig. 7, the snake's head ( 1 ) is compared with the head of a sea horse (2), whose size is 2-3 times less. One can recall a long list of similar cases.

The snake's head is topped with an indistinct bright mass that resembles a tuft that is found in numerous Earth species. To the left of the 'eye,' there is a slightly elongated spot (Fig. 5) that changes its shape in the successive shots of Fig. 6. By analogy with Earth 
organisms, one can assume that it is a hearing organ (acoustic phenomena on Venus were noted in [4]).

Fig. 6. Trea hages: $(-$ a fragment from panorama BW-1, series 6 ; (2) - a fragment from panorap $\mathrm{V}-1$, so $9 / 12$ and (3) - panorama BW-3, series $9 / 13$. Comparing the fragments makes it po ble to ry some small relative moves of the snake's parts during the 1.5 hours.

Th ake's head is topped with an indistinct bright mass that resembles a tuft that is found in n merous Earth species. To the left of the 'eye,' there is a slightly elongated spot (Fig. 5) that changes its shape in the successive shots of Fig. 6. By analogy with Earth organisms, one can assume that it is a hearing organ (acoustic phenomena on Venus were noted in [4]). In Figs. 5 and 6, above the body of the honeycomb entity (2), one can see a "crest' (3) that is approximately $4 \mathrm{~cm}$ long and is composed of separate elements that are bounded with an arc. In blinking, it was the crest that noticeably changed its position in the successive shots (shifting relative to a small dark area of the surface neighboring the 'crest' on the left). The combined images can slightly deteriorate precisely because of their being stacked when the crest is chosen as the pilot detail for stacking (or, alternatively, the crest 
could become fuzzy in other stacking options). The head (1) and the bulging part (2) of the snake's body (Fig. 5) also shift somewhat when blinking the successive shots shown in Figs. 4 and 6. It appears that the snake ends with a long and broad 'tail' (4) (see Fig. 5), which butts on the right into a bright triangular rock fragment. As far as one can judge, the small bright detail that protrudes on top can be referred to as the 'tail' as well because, in blinking, the detail successively shifts to the left along the tail. Left from the protruding detail, there are two small dark spots. A slight change in the tail position can also be traced by the relative change in the position of the two spots mentioned above. One cannot see any other spots on the tail. However, the shifts in the position of the snake's parts can hardly be noticed without blinking.

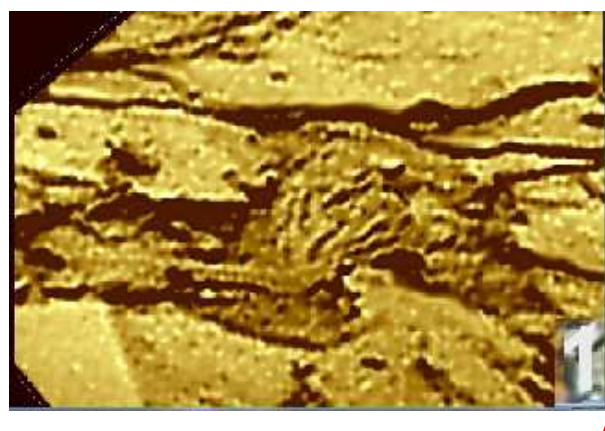

Fig. 7. The snake's image (1) and the image of a sea ho actual sizes are quite

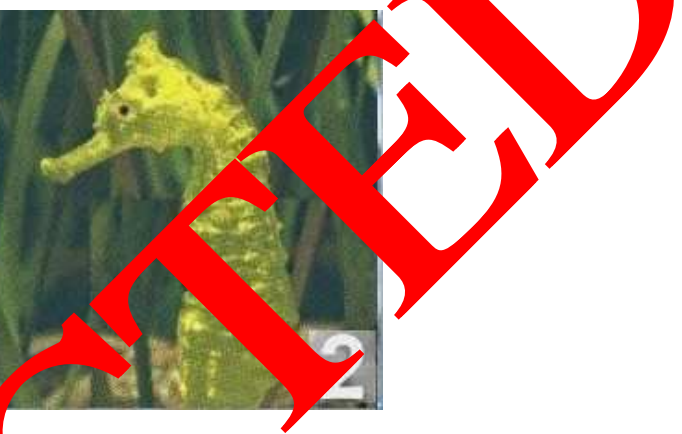

(2) with a s"milar shape of the head. The cerent.

The fragments of the VENERA-14 $p$ hor ar ander consideration are low noise, which offers a chance, in a number of ases, to arrive at distinct images even on the basis of a single panorama. The onc omb sul ace of the snake's body with its regular spots stands out so strongly against bac ground the surrounding stone plates that the entity cannot possibly be referred as oject.

Moreover, the snal can be in ded into the set of the most interesting findings of the Venus fauna because se ceeded in cording its successive motions. An interesting object called a 'dove' is als nota although one cannot identify the details of its structure. It is close to the sna' e, but that pro ity could be accidental. However, Earth beings of a smaller size can be $f$ and in any cases to accompany larger ones and consume food wastes of the latter. The est. 'ed sma shifts of the 'snake' (and the 'dove') once again reveal that the style of 'ysica tivity of the hypothetical Venus fauna is very slow, which might be asso lated ith som nergy restrictions, as was suggested in [4,5]. At the same time, a more che $r$ con for hat observation might stem from the physical properties of the very mater f whrm the Venus organisms are composed.

As noted repeatedly $[3,4,10,12]$, no liquid water can occur on the Venus surface; therefore, s,ch organisms must rely on something else instead. Resins, for example, feature slow plasticity. An entity that is made of such a viscous material requires some noticeable time for any change in its shape. Can it be that the reason behind the tardiness of the Venus fauna is the properties of this viscous media?

The 'snake' and the 'dove' are not the only unusual objects in the VENERA-14 panoramas. Fig. 8 shows an area that is close to the snake. Above the plate that is, in its turn, above the snake, there is a larger object of some periodic structure and, naturally, of unclear origin. 


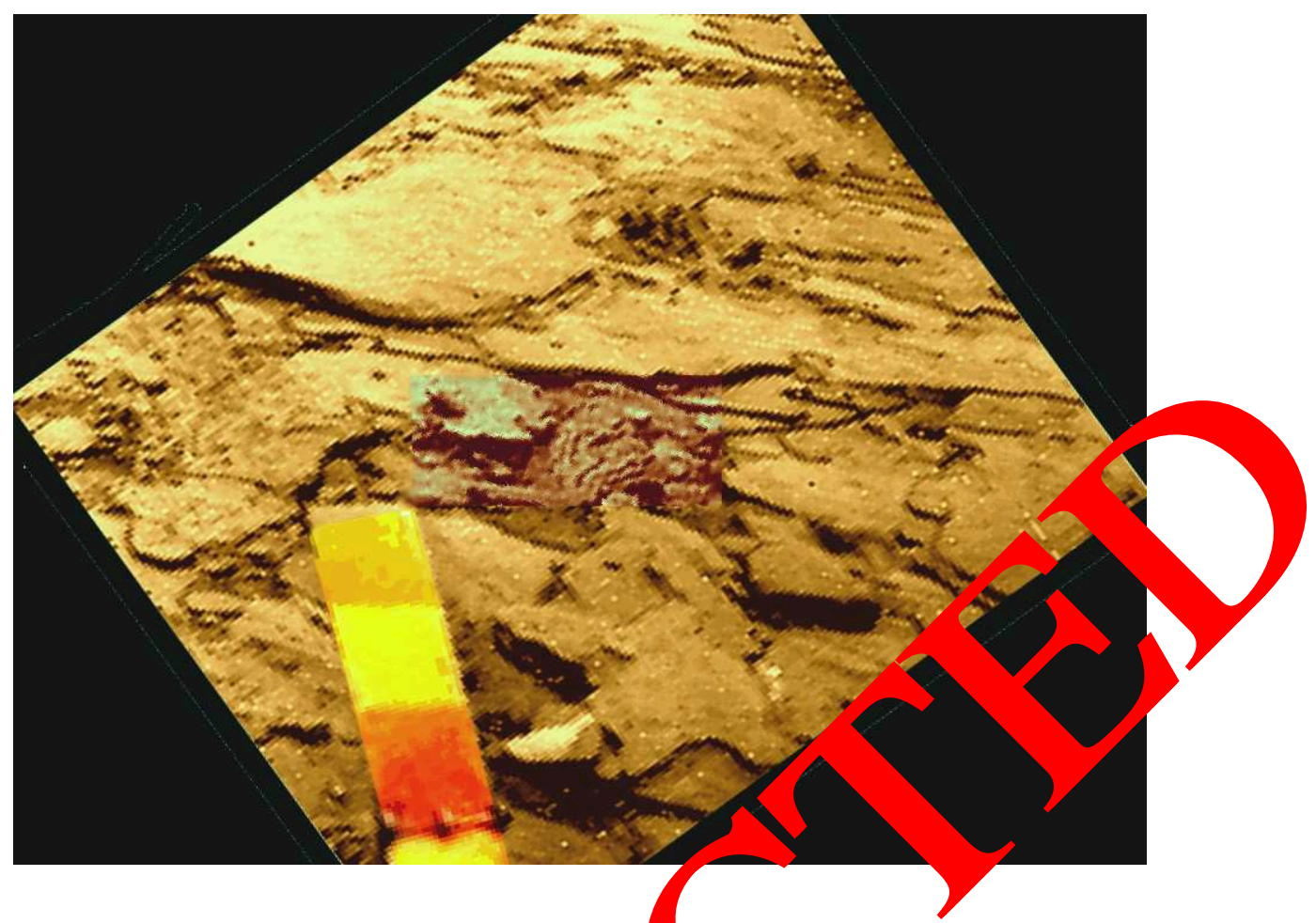

Fig. 8. A color sector of the VENERA-14 panorama w the 'snake' thd other objects, demanding further research. Compared with the stone plates, the snal as a darke tone (which is not the case for the green tint pictures). The green tint of thenlate with n top could be a result of the

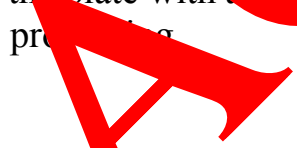

The object rises above the atrat and its ather complicated structure could be difficult to associate with a geological natio . In the $t \rho$ part of Fig. 8, at a left angle and in a round depression, one can see a righ a regular symmetric radial structure that resembles another object hat has a ilar structure in the VENERA-13 panoramas, which is close to the 'scorpion

\section{CONCLY IONS}

Th-analys f the ENERA-14 panoramas made it possible to reveal an object called a ' $\mathrm{s}$ ake at is $\hat{a}_{1}$ ximately $40 \mathrm{~cm}$ in length and possesses undeniable terramorphic fea. s. I ne snotty, honeycomb surface of the snake's body is clearly different from the backg. a, II surrounding stone plates. The 'snake' can be referred to as one of the most interestì sindings among the hypothetical Venus fauna. Another finding, a 'dove,' is of interest as -ell, although because of the distance and small size, the details of its structure can hardly be identify. The snake and the dove were found to move, although slowly, which confirms once again to the data of $[2-6,13]$ on the slow pattern of physical activity of the Venus fauna (as compared with the Earth fauna), which could be due to its energy constraints.

However, there might be a different, no less plausible reason behind that, inasmuch as water, an active medium in Earth organisms, does not exist on Venus in its liquid form; therefore, there should be something else instead. Unlike water, the active medium that is the basis of the Venus fauna can feature slow plasticity. Resins, for example, possess such a property. This medium requires substantial time for any change in its shape. With respect to 
the terramorphic features of the Venus fauna [4-6,12-14], in case they are confirmed, they would point at exceptionally important general laws of a living nature, yet undiscovered by science, occurring at absolutely different physical conditions, including the high-temperature, oxygen-free Venus environment or the settings of some types of exoplanets. A number of VENERA-14 panoramas are low-noise, which gives a chance, as it is shown in the paper, to obtain clear images even on the basis of a single shot. On the whole, the VENERA-14 panoramas contain a sizeable number of uncommon objects that demand further research.

\section{References}

[1] A. S. Selivanov, Yu. M. Gektin, M. A. Gerasimov, et al., Kosmich. Issle 21 1983) 176-182.

[2] L. V. Ksanfomality, Doklady Physics 57(9) (2012) 367-372.

[3] L. V. Ksanfomality, Solar System Research 46(1) (2012) 41-

[4] L. V. Ksanfomality, Doklady Physics 57(12) (2012) 497 - 01.

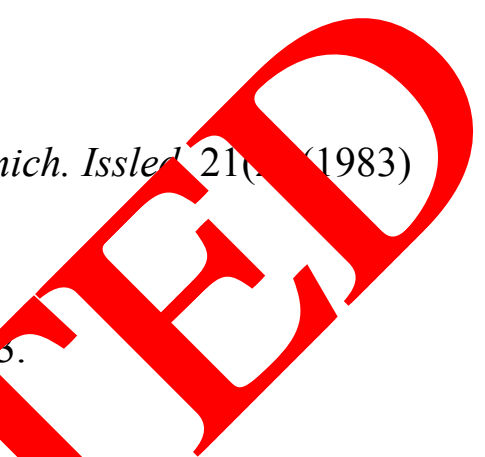

[5] L. V. Ksanfomality, International Journal of Astrono and Astr vics 3 (2013) 57-79.

[6] L. V. Ksanfomality, International Letters of Che istry, Physics and Astronomy 10 (2013) 76-89.

[7] K. P. Florensky, A. T. Basilevsky, V. P. Kruchkov, 340-350. [8] E. N. Lasarev, J. F. Rodionova, V. V. Sher her so, Ius Relief Map, Moscow State Univ.
Press. 2012.

[9] A. L. Kemurjian, P. N. Bro ky, V. I. Grom ,et al., Kosmich. Issled. 21(3) (1983) 323-330.

[10] L. V. Ksanfomality oklady A, ics 58(5) (2013) 196-199.

[11] B. E. Moshkin, (.P. \ omov, D... Moroz et al., Kosmich. Issled. 21(2) (1983) 236-245.

[12] L.V. Ks foma voklany Physics 58(4) (2013) 395-400.

[13] L.V. Ksan ality, A vances in Zoology and Botany 1(2) (2013) 25-29.

[14] L.V. anfom. Doklady Physics 58(5) (2013) 204-206.

(Received 04 November 2013; accepted 12 November 2013) 